\title{
Guest Editors' Introduction: Special Section on InfoVis
}

\author{
Daniel A. Keim, Member, IEEE Computer Society, Tamara Munzner, and \\ Stephen C. North, Senior Member, IEEE
}

$\mathrm{T}$ HREE papers in this issue of TVCG are expanded versions of ones presented at the IEEE Symposium on Information Visualization (InfoVis) 2003. These examples convey the breadth of work in the InfoVis community, attacking practically motivated problems from sophisticated mathematical and scientific perspectives. They demonstrate an interaction technique for navigation, a mathematically well-founded approach to the difficult problem of dimensionality reduction, and an evaluation of a particular channel of visual perception.

"A Model for Smooth Viewing and Navigation of Large 2D Information Spaces" by Jarke van Wijk and and Wim Nuij won the InfoVis Best Paper Award. They consider the problem of combining panning and zooming for moving around in large information spaces. Applying techniques from differential geometry, they derive a closed form solution and provide empirical values for its parameters. (In their presentation at InfoVis, they polled the audience to find the most pleasing trade off.) Interestingly, their work also settles an open question proposed by Mackinlay, Card, and Robertson in their 1990 SIGGRAPH paper, "Rapid Controlled Movement through a Virtual 3D Workspace."

"Robust Linear Dimensionality Reduction" by Liran Carmel and Yehuda Koren is a fine example of assisting data analysis through visualization. The authors present several algorithms for projecting multidimensional data onto lower spaces via linear transformations, extending the basic PCA (principal components analysis) technique to handle weights on the distances between points. This makes it convenient, for example, to incorporate categorical labels and to downweight the effects of outliers in the data.

"Conveying Shapes with Texture: Experimental Investigations of Texture's Effects on Shape Categorization Judgments" by Victoria Interrante, Sunghee Kim, and Haleh Hagh-Shenas describes detailed user studies investigating the effectiveness of novel texture synthesis techniques previously published in TVCG. The question of how texture affects the perception of shape in computer graphics is subtle. The authors introduce an experimental methodol-

- D.A. Keim is with the Department of Computer and Information Science, University of Constance, 78457 Konstanz, Germany. E-mail:keim@informatik.uni-konstanz.de.

- T. Munzner is with the Department of Computer Science, University of British Columbia, 2366 Main Mall, Vancouver BC V6T 1Z4 Canada. E-mail: tmm@cs.ubc.ca.

- S.C. North is with ATET Shannon Laboratory, Information Visualization Research, 180 Park Ave. Bldg. 103, Florham Park, NJ 07932-0971. E-mail: north@research.att.com. ogy and show that, for a certain set of shape recognition problems, textures with patterns oriented along the principle direction of curvature increased the accuracy of shape category judgments as long as the view is from an oblique direction. InfoVis encompasses studies of the synthesis and perception of information that is not intrinsically geometric and this work is relevant to a broad range of visualization applications.

\section{Daniel A. Keim Tamara Munzner Stephen C. North Guest Editors}

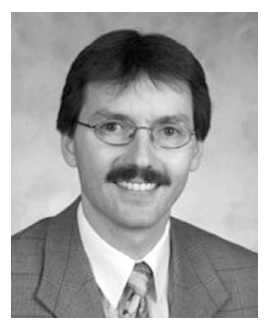

Daniel A. Keim received the PhD degree in computer science from the University of Munich in 1994. He works in the area of information visualization and data mining. In the field of information visualization, he developed several novel techniques which use visualization technology for the purpose of exploring large databases. $\mathrm{He}$ has published extensively on information visualization and data mining; he is an editor of the IEEE Transactions on Visualization and Computer Graphics and the Information Visualization Journal. He has been with the Computer Science Department at the University of Munich, the Computer Science Department at the Martin-Luther-University Halle, and is a full professor in the Computer Science Department at the University of Constance. Recently, he worked at AT\&T Labs, Florham Park, New Jersey. He is a member of the IEEE Computer Society.

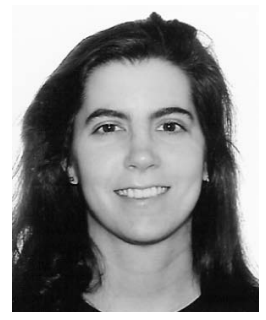

Tamara Munzner received the $\mathrm{PhD}$ degree from Stanford University in 2000. She has been an assistant professor at the University of British Columbia Department of Computer Science since 2002. Her current research interests are information visualization, graph drawing, dimensionality reduction, and interactive computer graphics. She was a research scientist from 2000 to 2002 at the Compaq Systems Research Center in California. She was on the technical staff of The Geometry Center, a mathematical visualization research group at the University of Minnesota, from 1991 to 1995.

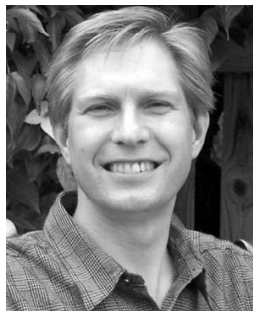

Stephen C. North received the PhD degree in computer science from Princeton University in 1986. He is head of Information Visualization Research at AT\&T Labs, a group that studies novel interactive displays and high-performance graphics for network visualization in the AT\&T Infolab. His background is in software visualization, applied computational geometry, and the design of reusable software. He is one of the authors of graphviz, a widely used collection of open source programs for drawing and interacting with graph layouts. His other current technical interests include dynamic and large scale graph visualization, spatial data transformation, and high-performance visualization systems. He is a senior member of the IEEE and a member of the ACM. 